\title{
Characteristics of the 2nd Harmonic ECR Micro Plasma Sources by Using PIC/MCC Simulations
}

\author{
M. Radmilović-Radjenović ${ }^{a, *}$, B. Radjenović ${ }^{a}$ And P. BeliČev ${ }^{b}$ \\ ${ }^{a}$ Institute of Physics, University of Belgrade, Pregrevica 118, 11080 Belgrade, Serbia \\ ${ }^{b}$ Vinča Institute of Nuclear Science, University of Belgrade, 11001 Belgrade, Serbia
}

(Received January 11, 2012; in final form March 9, 2012)

\begin{abstract}
In this paper we have presented our simulation studies of $2.4 \mathrm{GHz}$ microwave plasma production under the electron cyclotron resonance with an idea to expand the plasma generation conditions into the much lower pressure range and much shorter gap length. As the first for this purpose, we have focused on the influences of applied magnetic field, gas pressure and gap length on the breakdown and maintenance of plasmas. Calculations were performed by using a one-dimensional particle-in-cell/Monte Carlo collisions code with three velocity components. The obtained simulation results are in a good agreement with the available experimental data providing an insight into the resonant electron acceleration for ECR condition and the resonant electron confinement for the $2 \mathrm{nd}$ harmonic ECR. In addition, analytical expressions for the breakdown voltage and the trapping field have been derived.
\end{abstract}

PACS: 52.25.Os, 52.65.-y

\section{Introduction}

Electron cyclotron resonance (ECR) microwave discharge plasmas have been widely used for various applications in plasma-aided processing due to their high degree of ionization, low and controllable plasma sheath potential and electrodeless operation [1]. Recently much attention has been paid to microscale science and technology [2], so generation of low pressure microplasma has also emerged as a very important topic [3]. Generation of microplasmas with several $100 \mu \mathrm{m}$ size at pressure below 1 Torr, ECR is very useful because not only for its electron acceleration effect, but also the electron confinement effect [4].

Many diagnostics techniques have been performed for studying breakdown conditions in ECR plasmas. Lax et al. [5] have observed a decrease of the breakdown electric field strength when the ECR conditions were satisfied. Hayashi et al. [6] have reported the breakdown condition for an rf discharge in low pressure gases in the presence of a strong magnetic field. On the other hand, some numerical investigations have also been made. Hussein and his co-workers [7] have used a one-dimensional code to clarify the effect of collisions on ion dynamics. Choi et al. [8] have developed a hybrid electron fluid-particle ion model for treating the microwave discharge characteristics. Later, Zhong et al. [9] have described Monte Carlo simulations of argon ion transport in the downstream region of ECR plasmas.

\footnotetext{
* corresponding author; e-mail: marija@ipb.ac.rs
}

In this paper, we have studied low pressure ECR microplasma. As the first step, we have studied the gaseous breakdown conditions for microwave power in the presence of various discharge conditions for breakdown, non-trapping and electron ionization threshold by using a one-dimensional particle-in-cell/Monte Carlo (PIC/ MCC) collision code [10] adjusted to simulate an ECR plasma.

\section{Trapping condition}

Calculation model used in this paper is similar to that described in Ref. [6] and shown in Fig. 1. In derivation the expression for the maximum trapping electric field defined as the maximum electric field required to confine the electrons in the gap space, we shall start from the expression for the electron orbit in the $x$ direction in a vacuum

$$
x=\frac{e E}{\left(\omega_{\mathrm{ce}}^{2}-\omega^{2}\right) m}\left(\frac{\omega}{\omega_{\mathrm{ce}}} \sin \left(\omega_{\mathrm{ce}} t\right)-\sin (\omega t)\right),
$$

with the phase and constant of integration set to zero. If $x$ is greater than the gap length the initial electron is lost and it is not possible for breakdown to occur. The maximum amplitude in the $x$ direction is

$$
E=\frac{d\left(\left(\frac{\omega_{\mathrm{ce}}}{\omega}\right)^{2}-1\right) \omega^{2} m}{2 e} \frac{1}{\left(\frac{\sin (\omega t)}{\left(\frac{\omega_{\mathrm{ce}}}{\omega}\right)}-\sin (\omega t)\right)},
$$

where $x=(b-a) / 2$. The trapping condition can be determined by using Eq. (2) as the electric field where the maximum amplitude in the $x$ direction is equal to 

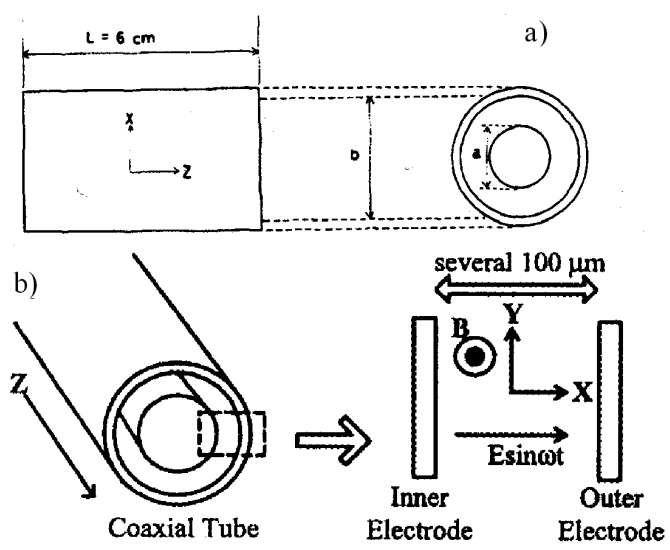

Fig. 1. (a) Structure of the coaxial tube with discharge volume $L \pi\left[(b / 2)^{2}-(a / 2)^{2}\right]$ and (b) calculation model.

half of the gap length.
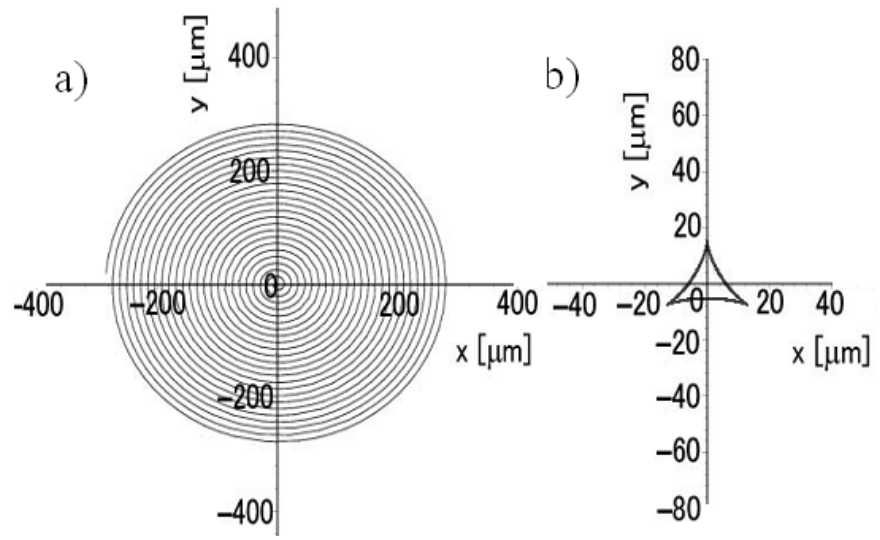

Fig. 2. The difference in the electron orbits between: a) ECR $\left(\omega_{\text {ce }} / \omega=1\right)$ and b) 2nd harmonic ECR conditions $\left(\omega_{\mathrm{ce}} / \omega=0.5\right)$.

As illustrated in Fig. 2, electron cyclotron resonance generally occurs at $\omega_{\text {ce }} / \omega=1$. In this condition electrons are continuously accelerated and the radius of the electron orbit increases with time. On the other hand, at $\omega_{\text {ce }} / \omega=0.5$, displacements of the electrons are very small, suggesting that the electron confinement is better than that for usual ECR condition.

\section{Breakdown condition}

In order to obtain the breakdown criteria for high frequency discharges at low pressure under the influence of a strong magnetic field we have followed the procedure established by Hayashi et al. [6]. We combine a balanced equation of electron generation and loss

$$
Z\left[\pi\left(\frac{b}{2}\right)^{2}-\pi\left(\frac{a}{2}\right)^{2}\right] L n_{\mathrm{c}}
$$

$$
=\left\{(\pi b L+\pi a L) v_{\mathrm{e} x}+\left[\pi\left(\frac{b}{2}\right)^{2}-\pi\left(\frac{a}{2}\right)^{2}\right] v_{\mathrm{e} x}\right\} \frac{n_{\mathrm{e}}}{2},
$$

with an equation that describes the effective electron thermal velocity in the magnetic field

$$
v_{\mathrm{e} x}=v_{\mathrm{e} y}=\frac{v_{\mathrm{e} z}}{\sqrt{1+\left(\frac{\omega_{\mathrm{ce}}}{v}\right)^{2}}},
$$

and an equation for the power consumption of the electrons

$$
Z e V_{i}=e \operatorname{ERe}\left(v_{a x}\right),
$$

where $Z$ represents the ionization frequency, $L$ is the length of the discharge space in the $z$ direction defined by the magnetic field direction and $b$ and $a$ are the diameters of the outer and inner electrodes, respectively, as can be seen from Fig. 1. Components of the thermal velocity along the $x, y$, and $z$ directions are denoted by $v_{\mathrm{e} x}$, $v_{\mathrm{e} y}$ and $v_{\mathrm{e} z}$, respectively, and collision frequency between neutral particles of the gas and electrons is given by $\nu$. $V_{i}$ is the ionization voltage and $\operatorname{Re}\left(v_{a x}\right)$ is the label for the real part of the velocity of electrons accelerated by microwave. Combining Eqs. (3)-(5) we have obtained expression for the minimum breakdown field defined as the minimum electric field required to start discharge:

$$
\begin{aligned}
E & =\left(2 m \omega^{2} V_{i}\left[\left(\frac{\nu}{\omega}\right)^{2}+\left(1+\frac{\omega_{\mathrm{ce}}}{\omega}\right)^{2}\right]\right. \\
& \times\left[\left(\frac{\nu}{\omega}\right)^{2}+\left(1+\frac{\omega_{\mathrm{ce}}}{\omega}\right)^{2}\right]\left\{\frac{1}{2 L}+\frac{1 / d}{\left[1+\left(\omega_{\mathrm{ce}} / \nu\right)^{2}\right]^{1 / 2}}\right\} \\
& \left.\times \frac{v_{\mathrm{e}}}{3^{1 / 2} e \nu}\left[1+\left(\frac{\omega_{\mathrm{ce}}}{\omega}\right)^{2}+\left(\frac{\nu}{\omega}\right)^{2}\right]\right)^{0.5}
\end{aligned}
$$

\section{Simulation technique}

We are witnessing that the simulations become very powerful tool in all areas of science. The simulation code used in this study is a one-dimensional particle-in-cell (PIC) code with Monte-Carlo treatment of collisions (see, for example, Ref. [10]). Calculations were performed by using well established cross-section data for argon [11]. Simulation conditions were based on the experimental conditions published in Ref. [3]:gap size of $3.5 \mathrm{~mm}$ and the gas pressure ranging from 0.006 Torr to 0.1 Torr. The inner radius of the cylindrical electrode is $2.5 \mathrm{~mm}$

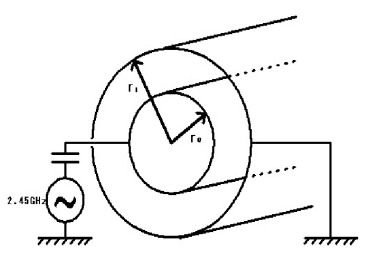

Fig. 3. Schematic diagram of the simulation system. 
connected with $2.45 \mathrm{GHz}$ microwave generator, while the outer electrode radius is $6 \mathrm{~mm}$, as shown in Fig. 3. The upper electrode is grounded. Such a reactor is asymmetric since the grounded electrode is larger than the powered one.

\section{Results and discussion}

Figure 4 shows the magnetic field required to minimize the breakdown electric field for a given gas pressure. Our simulation results (open symbols) are in a good agreement with the results of measurements carried out by Kumamoto et al. [3] (solid symbols). The lower values obtained in the simulations as compared to the experimental data are attributed to differences between the simulation and experimental conditions. Although the same gap length is used in both cases, the temperature and electrode materials used in the experiments are unknown. Differences in temperature and surface conditions are believed to cause the differences observed in [3] between simulations and experimental results. Finally, results of analytical expressions (6) derived in this paper (curves) shows similar trends as experimental and simulation results having in mind that developed model of ECR source is very simple model.

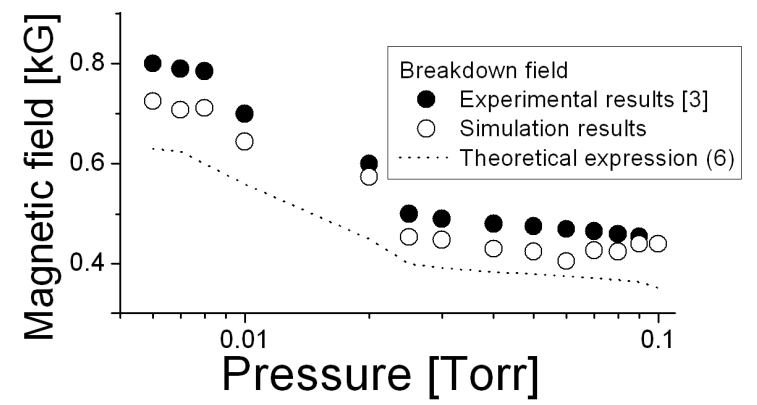

Fig. 4. The dependence of the magnetic field on the gas pressure. Simulation results (open symbols) are compared with the experimental results taken from Ref. [3] (solid symbols) and theoretical prediction obtained by using Eq. (6) (dot curve).

A satisfactory agreement between simulation (open symbols), experimental (solid symbols) and theoretical results (curve) can be also noticed in Fig. 5 which displays maximum trapping magnetic field. Both figures shows that plasma occurred at the "breakdown magnetic field". Simulations were performed for the electric field defined by Eq. (2), where $E$ is set to the highest magnitude that still prevents electrons from transiting the electrode gap with increasing magnetic field, the plasma vanished at the "maximum trapping magnetic field". The range from the breakdown magnetic field to the maximum trapping magnetic field can be considered as the plasma discharge region.

Finally, Fig. 6 demonstrates that electron energy distribution function (EEDF) for the ECR plasma lacks the

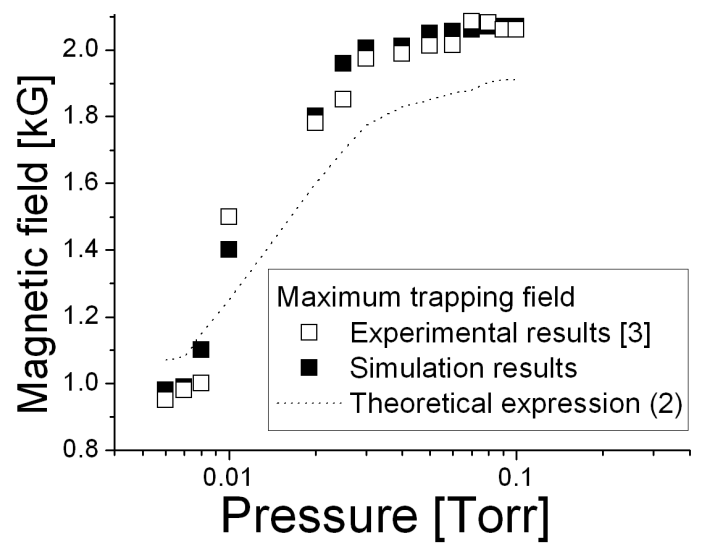

Fig. 5. The magnetic field versus the gas pressure. Simulation results are shown by solid symbols, while open symbols correspond to the experimental results taken from Ref. [3]. Theoretical prediction obtained by using Eq. (2) are represented by dot curve.

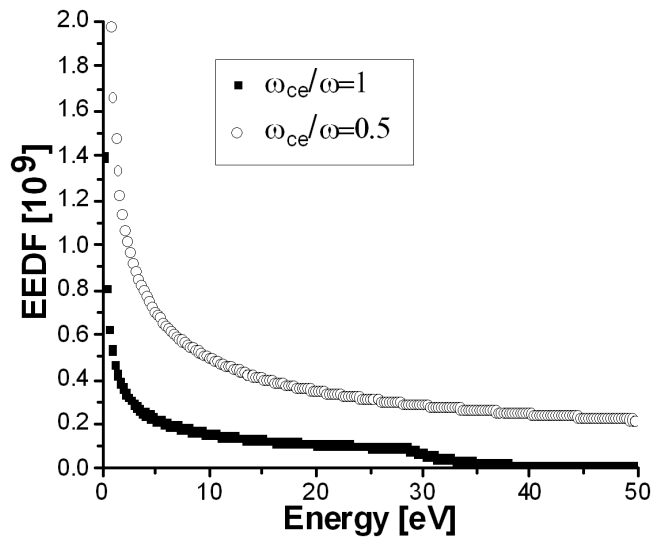

Fig. 6. Electron energy distribution function (EEDF) for $\operatorname{ECR}\left(\omega_{\mathrm{ce}} / \omega=1\right)$ and 2 nd harmonic ECR conditions $\left(\omega_{\mathrm{ce}} / \omega=0.5\right)$

high energy tail due to the fact that the hottest electrons exceed the boundary of the system and are lost. Simulation results demonstrate the resonant electron acceleration for ECR condition and the resonant electron confinement for the 2nd harmonic ECR. In other words, the electron confinement for the 2nd harmonic ECR plasma is better than that for ECR plasma.

\section{Conclusions}

In this paper, the characteristics of ECR and 2nd harmonic ECR plasmas have been investigated by using a one-dimensional PIC/MCC code. We were focused on various discharge conditions for breakdown and electron trapping fields. In addition, simple theoretical model has been applied and expressions for the breakdown voltage and the trapping field have been derived. The obtained simulation results show a satisfactory agreement with the available experimental data. Results achieved by using 
described simple theoretical model show similar trends as compared to the results of measurements and the simulation results. These results demonstrate the resonant electron acceleration for ECR condition and the resonant electron confinement for the 2nd harmonic ECR.

\section{Acknowledgments}

The authors acknowledge financial support of the Ministry of Education and Science of the Republic of Serbia O171037.

\section{References}

[1] M. Matsushita, Y. Matsuda, H. Fujiyama, Thin Solid Films 435, 285 (2003).

[2] T. Ito, K. Terashima, Appl. Phys. Lett. 80, 2854 (2002).

[3] M. Kumamoto, H. Inoue, M. Matsushita, H. Fujiyama, Thin Solid Films 475, 124 (2005).
[4] T. Shigemizu, N. Ohno, K. Fujiyama, Mater. Sci. Eng. A 139, 312 (1991).

[5] B. Lax, W.P. Allis, S.C. Brown, J. Appl. Phys. 21, 1297 (1950).

[6] Y. Hayashi, T. Kato, S. Takeda, Trans. IEEE Jpn. A 105, 605 (1985).

[7] M.A. Hussein, G.A. Emmert, N. Hershkowitz, R.C. Woods, J. Appl. Phys. 72, 1720 (1992).

[8] N.H. Choi, W.H. Koh, N.S. Yoon, H.B. Pard, D.I. Choi, IEEE Trans. Plasma Sci. 23, 617 (1995).

[9] X.X. Zhong, J.D. Wu, C.Z. Wu, F.M. Li, J. Appl. Phys. 83, 5069 (1998).

[10] C.K. Birdsall, IEEE Trans. Plasma Sci. 19, 65 (1991).

[11] M. Radmilović-Radjenović, B. Radjenović, Plasma Sources Sci. Technol. 16, 337 (2007). 